and co-workers (Fig. 1b). The encoding of two letters in the same space shows beyond a doubt that quantum interference is necessary to form the holographic image. In a sense the image is three-dimensional: the two spatial dimensions of the surface plus a third dimension of energy.

Information density is always one of the key issues when data is encoded. The higher the density (or the smaller the writing), the better: you don't want to lug around something the size of a suitcase for one page of text. The calculated information density in the Stanford experiments depends on whether you include the array of molecules that surround the read-out area. The Stanford group quotes a figure of $20 \mathrm{bits} \mathrm{nm}^{-2}$ for the information density in some of their experiments, although this figure is based on the read-out area (which is typically about $4 \times 5 \mathrm{~nm}^{2}$ ) rather than the area occupied by the carbon monoxide molecules (which is approximately $17 \times 17 \mathrm{~nm}^{2}$ ). It may seem pedestrian to use letters of the alphabet, but they are just as good a target shape as anything else, and of course we are familiar with them and can see that they are deliberate structures. If you can create the letter ' $S$ ' and the letter ' $U$ ' in the same space, the chances are good that you can also create 100 or so other distinct shapes in the same space.
Nobody is going to be mass-producing high-density messages encoded in carbon monoxide molecules on copper surfaces given the low-temperature and high-vacuum conditions required, never mind the length of time it takes to arrange the molecules so that the letters appear holographically, or the CPU time needed to work out where to place the molecules. What Manoharan and co-workers demonstrate is the possibility of going beyond the limits of information density that seem to be imposed in this approach by the finite wavelength of the electrons and the closest possible spacing between molecules. Indeed, they achieve an information density that is $50 \%$ greater than the density that can be achieved by using the molecules themselves in conventional writing - if you can call writing molecule-by-molecule conventional! This is made possible in part by the same phenomena that have made sub-wavelength imaging possible in optics: near-field effects. As long as the sources or scatterers of the waves in question have a physical dimension that is much less than the wavelength, the wave field will carry sub-wavelength detail near the sources or scatterers that may be used in near-field imaging. Another part of the story is the energy dependence of the scattering, which is exploited to encode more information in the same area.
The concept of quantum holography pops up from time to time, but it has not caught on as far as I know. Typing 'quantum holography' in Google reveals one or two credible uses of the term, and a long list of less-credible uses, including a claim from Ed Mitchell, the Apollo 14 astronaut, that "Intuition, telepathy, clairvoyance and many similar information phenomena seem to be easily explained by means of the non-local quantum hologram." I know what he means, and after all he has seen things the rest of us have not. This time, however, I think the term will stick, because Manoharan and this talented team have finally demonstrated quantum holography for real.

Eric J. Heller is in the Department of Physics and Department of Chemistry, Harvard University, Cambridge, Massachusetts 02138, USA. e-mail:heller@physics.harvard.edu

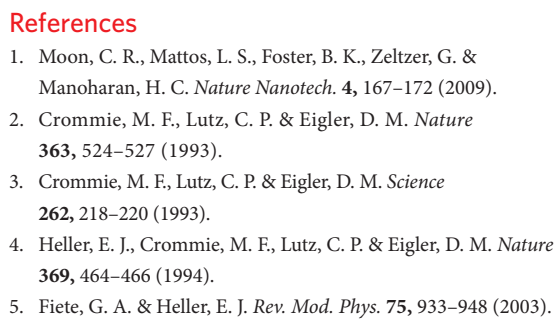

References 63, 524-527 (1993)

262, 218-220 (1993).

369, 464-466 (1994)

5. Fiete, G. A. \& Heller, E. J. Rev. Mod. Phys. 75, 933-948 (2003).

\title{
Probing superconductivity at the nanoscale
}

Many properties of metals, semiconductors and superconductors are determined by the shape of their Fermi surface the surface in momentum space that separates filled electron states from unfilled states at absolute zero temperature. In superconductors, a detailed knowledge of the Fermi surface is essential for an understanding of their physical properties, including their ability to conduct electricity with resistance. However, most techniques for measuring the Fermi surface of superconductors only produce spatially averaged data, rather than information on how the surface changes on nanometre length scales.

In recent years a number of groups have used scanning tunnelling microscopy (STM) to study the properties of hightemperature cuprate superconductors in greater detail, including spatial variations in the superconducting energy gap and the influence of dopant atoms. Now, Eric Hudson and colleagues at MIT, Nagoya University and the Brookhaven National
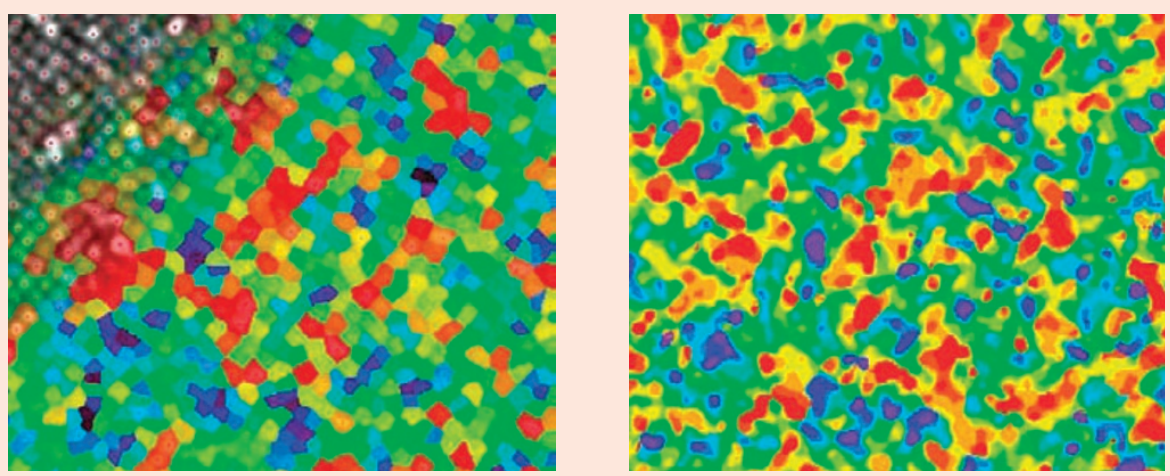

Laboratory have extended a technique called Fourier-transform STM to image variations in the Fermi surface of bismuth-based cuprate superconductors at the nanoscale (Nature Phys. 5, 213-216; 2009).

STM can be used to measure the local density of states for electrons which is related to conductance - as a function of energy and position in two dimensions. These maps can then be Fourier transformed to learn more about the Fermi surface. Hudson and co-workers found that the Fermi surface changes on the nanoscale, as these images of the conductance (left) and energy gap (right) show. Each image is $60 \mathrm{~nm}$ across; red corresponds to a high conductance and a small gap. The nanoscale variations in the Fermi surface are thought to be due to variations in the distribution of dopants in the superconductor.

ADARSH SANDHU 


\section{Corrections}

In the News \& Views 'Probing superconductivity at the nanoscale' (Nature Nanotech. 4, 142; 2009), the penultimate sentence should have read: 'red corresponds to a high conductance and a small gap.'

Corrected in the HTML and PDF versions, after print: 7 October 2009.

In the News \& Views 'Crossing boundaries and borders' (Nature Nanotech. 1, 91-92; 2006), the third sentence of the second paragraph should have referred to Claire Berger.

Corrected in the HTML and PDF versions, after print: 7 October 2009. 